\title{
Bioinformation transformation: From ionics to quantum ionics
}

\author{
Xiqi Zhang ${ }^{1}$, Markus Antonietti ${ }^{4}$ and Lei Jiang ${ }^{1,2,3^{*}}$
}

In the neural system, a large number of neurons continuously produce and transmit electrophysiological signals, which communicate between neurons and brain regions. Naturally, bioinformation transformation depends on conversion from ionics to electronics, and electronics-based neural recording technologies (Fig. 1) have been extensively developed since the primitive voltage clamp in 1949 [1]. Then patch clamp technology was created by Neher and Sakmann in 1976 [2]. Afterwards, microfabricated silicon Utah-type microelectrode arrays were constructed in 1990 [3]. Recently, field effect transistors [4], nanowire field effect transistors [5], carbonbased electrodes [6], organic electrochemical transistors [7] and mesh electronics [8] have been designed for neural recording with improved performance. However, these electronics-based neural recording technologies according to the Hodgkin theory have made little progress in the field of neural signal research, which hinders the study of the human brain project, such as the failure of Blue Brain Project in Europe. In the traditional Hodgkin theory of neural signal transmission (Fig. 2a), the generation of the action potential is accompanied by disordered entropy-driven ion diffusion along the direction of the axon, which shows slow propagation velocity $\left(0.2-120 \mathrm{~m} \mathrm{~s}^{-1}\right)[9,10]$. Meanwhile, the ion diffusion process in the action potential conduction needs to consume large amounts of energy, which is not applicable to explain the rapid nerve signal transmission with a huge amount of data. In other words, the action potential has nothing to do with the actual instantaneous response of neural signal [11]. Therefore, there is an urgent need to develop a new technology to replace the traditional electronics-based technology.

Recently, we have found that the ion and molecule transport in ion channels of living systems has similar ordered superfluid process as that of liquid helium [1214]. This room temperature ionic and molecular superfluid is due to the size confinement of the ion/molecule

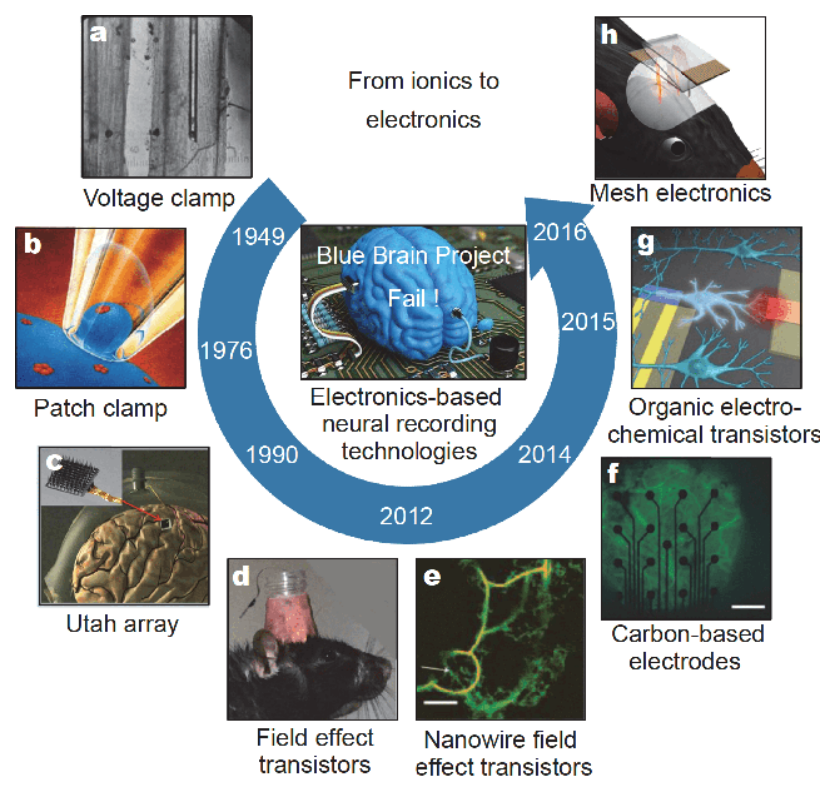

Figure 1 Electronics-based neural recording technologies: from ionics to electronics. (a) Voltage clamp; (b) patch clamp; (c) Utah array; (d) field effect transistors; (e) nanowire field effect transistors; (f) carbonbased electrodes; (g) organic electrochemical transistors; (h) mesh electronics. The blue curved arrow indicates time periods, and the image inside the curved arrow represents the Blue Brain Project in Europe, which proves the failure of electronics-based neural recording technologies in the brain science field.

\footnotetext{
${ }^{1}$ Key Laboratory of Bio-inspired Materials and Interfacial Science, Technical Institute of Physics and Chemistry, Chinese Academy of Sciences, Beijing 100190, China

${ }^{2}$ School of Future Technology, University of Chinese Academy of Sciences, Beijing 100049, China

${ }^{3}$ Key Laboratory of Bio-inspired Smart Interfacial Science and Technology of Ministry of Education, School of Chemistry, Beihang University, Beijing 100191, China

${ }^{4}$ Department of Colloid Chemistry, Max Planck Institute of Colloids and Interfaces, 14476, Potsdam, Germany

* Corresponding author (email: jianglei@iccas.ac.cn)
} 


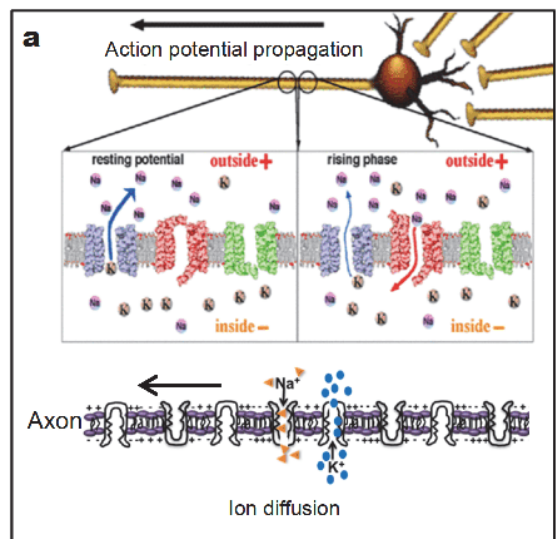

Ion diffusion in the Hodgkin theory

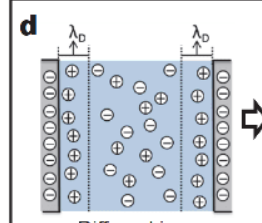

Diffused ions

THz spectra of diffused $\mathrm{K}^{+}$
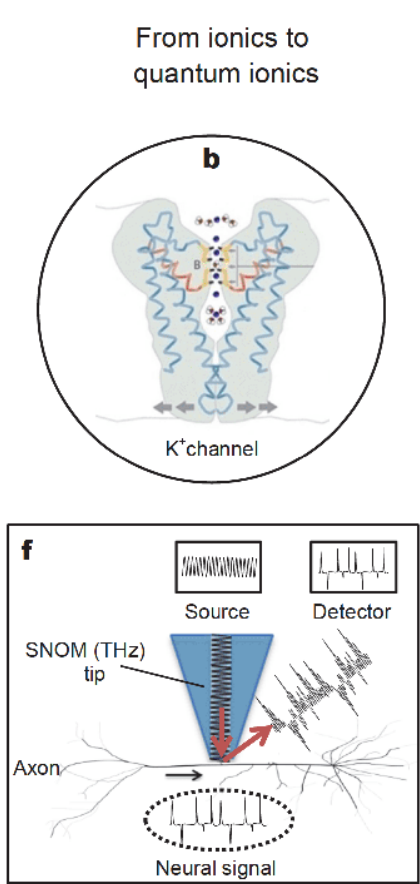

Biological quantum ionics technology

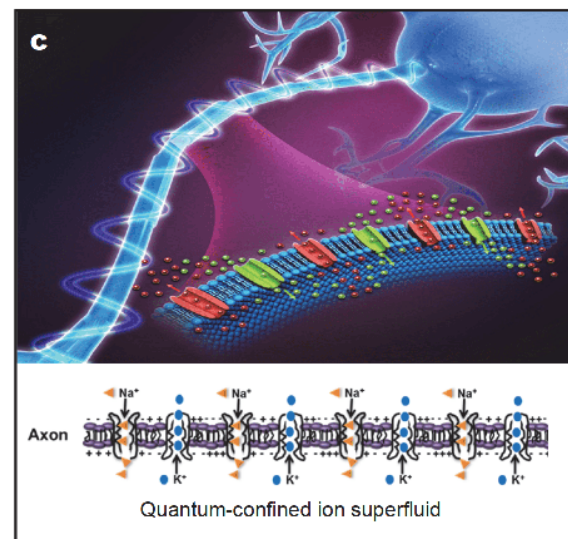

Quantum ionics-based neural signal

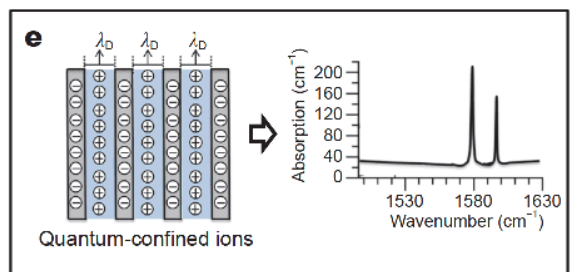

$\mathrm{THz}$ spectrum of quantum-confined $\mathrm{K}^{+}$

Figure 2 Biological quantum ionics technology: from ionics to quantum ionics. (a) Schematics of traditional neural signal transmission process based on the action potential theory, which generates through entropy-driven ion diffusion, consumes large amounts of energy and shows low transmission speed. (b) KcsA K ${ }^{+}$channel from Streptomyces lividans allows extremely fast ion transport ( $\sim 10^{7}$ ions/s), ultra-low energy consumption and ultra-low ionic resistance, demonstrating the existence of quantum-confined ion superfluid in $\mathrm{K}^{+}$channel. Reproduced with permission from Ref. [28]. Copyright 2004, Wiley-VCH. (c) Schematics of neural signal transmission process based on enthalpy-driven quantum-confined ion superfluid. (d) Schematic of diffused $\mathrm{K}^{+}$and $\mathrm{THz}$ absorption spectra of diffused hydrated $\mathrm{K}^{+}$, indicating $\mathrm{THz}$ absorption of diffused $\mathrm{K}^{+}\left(1 \mathrm{~mol} \mathrm{~L}^{-1}\right)$ locates at $\sim 160 \mathrm{~cm}^{-1}$ with a broad peak. Reproduced with permission from Ref. [21]. Copyright 2009, American Chemical Society. (e) Schematic of quantumconfined $\mathrm{K}^{+}$and $\mathrm{THz}$ absorption spectrum of quantum-confined $\mathrm{K}^{+}$. The schematic represents quantum-confined ion superfluid is formed in $\mathrm{K}^{+}$ channel with a channel size of Debye length $\left(\lambda_{\mathrm{D}}\right)$. The spectrum indicates $\mathrm{THz}$ absorption of quantum-confined $\mathrm{K}^{+}$shows two sharp peaks locate at $\sim 1580$ and $\sim 1603 \mathrm{~cm}^{-1}$. Reproduced with permission from Ref. [22]. Copyright 2016, American Association for the Advancement of Science. (f) Schematic of biological quantum ionics technology. Neural signals are studied by $\mathrm{THz}$ responses of quantum-confined ions in the biological channels. SNOM (THz) tip represents the tip of THz scanning near-field optical microscope. The source uses high frequency modulating THz light to stimulate the quantum-confined ions at their $\mathrm{THz}$ absorption peaks, and the neural signal is collected by a detector and obtained by processing.

channel in the organism. On the basis of biological ion channels, neural signal transmits in an instantaneous response type [11], the brain can process huge amounts of data [15], and the electric eels do not electrocute themselves [16], etc. In particular, $\mathrm{K}^{+}$channel in biological system allows extremely fast ion transport $\left(\sim 10^{7}\right.$ ions/s), ultra-low energy consumption and ultra-low ionic resistance [17]. In fact, ion and molecule flows in biological ion channels are regarded as quantum-confined superfluid, which are different from the ion diffusion process in neural networks (Fig. 2b). In this case, quantum states of ions and molecules could be bioinformation carriers with a huge amount of data (Fig. 2c) [11]. As the absorption of quantum states of ions and molecules is in the $\mathrm{THz}$ range [18], the $\mathrm{THz}$ light could be used as a tool to realize non-contact bioinformation detection [19]. Coincidentally, Liu [20] also conjectured the quantum states of ions and molecules in the nervous system are key factors for biological information, and the physical field of vertebrate neural signals should be in the $\mathrm{THz}$ range. We concretized that quantum states of ions and molecules are in the state of quantum-confined superfluid, and their absorption is in the $\mathrm{THz}$ range. Therefore, $\mathrm{THz}$ absorption and response could be used as a medium for neural signal detection. We further propose two approaches based on the bioinformation transformation from ionics to quantum ionics. The first one is to study the neural signals in biological systems by $\mathrm{THz}$ responses (Fig. 2). $\mathrm{THz}$ absorption spectra are employed to detect the signal of quantum-confined ion superfluid in biological ion 
channels. According to the Hodgkin theory, ions are in the diffused state, and the $\mathrm{THz}$ vibrational signals of diffused $\mathrm{K}^{+}\left(1 \mathrm{~mol} \mathrm{~L}^{-1}\right)$ locate at $\sim 160 \mathrm{~cm}^{-1}$ with a broad peak (Fig. 2d) [21]. In comparison, in the biological $\mathrm{K}^{+}$ channel, THz absorption of quantum-confined $\mathrm{K}^{+}$locates at $\sim 1580$ and $\sim 1603 \mathrm{~cm}^{-1}$ with two sharp peaks, which is obviously different from that of diffused $\mathrm{K}^{+}$ (Fig. 2e) [22]. Therefore, neural signals could be studied by $\mathrm{THz}$ responses of quantum-confined ions in the biological channels (Fig. 2f). THz scanning near-field optical microscope (SNOM) is a good candidate for simultaneous imaging and detection, which could achieve high temporal and spatial resolution [23]. Through the tip of SNOM, high frequency modulating $\mathrm{THz}$ light is used as a source to stimulate the quantum-confined ions in the axon at their $\mathrm{THz}$ absorption peaks, and the neural signal is collected by a detector and obtained by processing. This non-contact method for bioinformation detection could provide more information about neural signals, which would be superior to the traditional electronics-based neural recording technologies. However, the neural signal in a biological system is hard to detect because it is difficult to isolate the critical single channel or crystal from the biological environment, which needs new instrument and technology.

Another simple approach is to study the quantumconfined ion superfluid in artificial systems by $\mathrm{THz}$ responses, which could offer optimized parameters (e.g., frequency, amplitude, intensity, focus, and stimulation size) for the detection of neural signals in biological systems (Fig. 3). In artificial systems, subnanometer porous materials are used to mimic biological channels to investigate the transport of ions and molecules in biological systems. High frequency modulating $\mathrm{THz}$ light is used as a source to stimulate the quantum-confined ions in artificial channels, and the artificial neural signal is collected by a detector and obtained by processing (Fig. 3a). Therefore, subnanometer porous materials, including covalent organic frameworks (COFs) and metalorganic frameworks (MOF), could be used to simulate

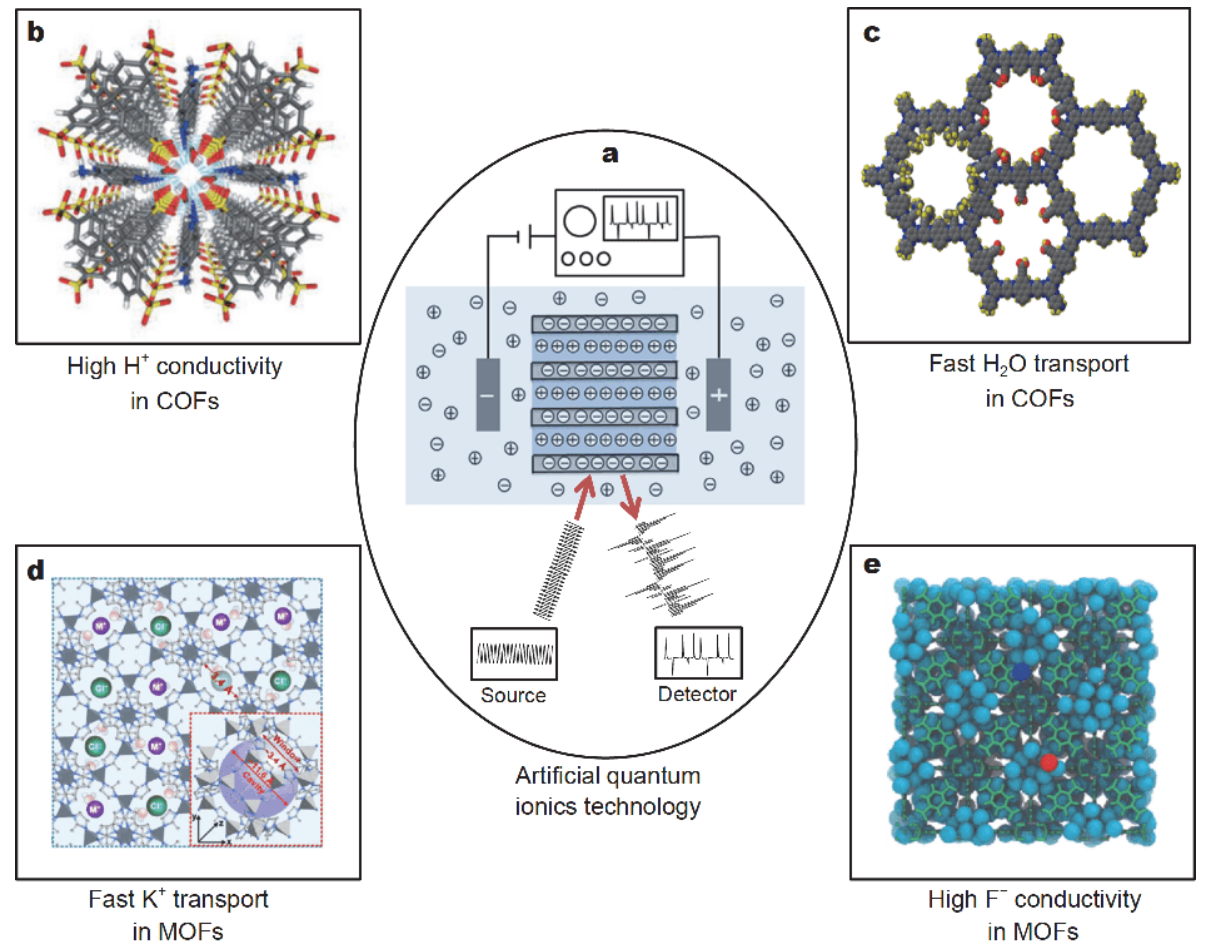

Figure 3 Artificial quantum ionics technology. (a) Schematic represents the quantum-confined ion superfluid study by THz responses in artificial systems, which could offer optimized parameters for the detection of neural signals in biological systems. Subnanometer porous materials are used to mimic biological channels, the source is high frequency modulating THz light, and the artificial neural signal is collected by detector and obtained by processing. (b) COFs show high proton conductivity of $2.2 \times 10^{-2} \mathrm{~S} \mathrm{~cm}^{-1}$ at ambient conditions. Reproduced with permission from Ref. [24]. Copyright 2018, Wiley-VCH. (c) COFs demonstrate both high-water permeation and ion sieving. Reproduced with permission from Ref. [25]. Copyright 2018, American Chemical Society. (d) MOFs with subnanometer pores show ultrafast selective transport of alkali metal ions. Reproduced with permission from Ref. [26]. Copyright 2018, American Association for the Advancement of Science. (e) MOFs with sub-1-nanometer channels demonstrate fast and selective fluoride ion conduction. Reproduced with permission from Ref. [27]. Copyright 2019, Nature Publishing Group. 
biological ion channels. The COFs derived from tetrakis (4-sulfophenyl)methane and p-phenylenediamine featured high proton conductivity of $2.2 \mathrm{~S} \mathrm{~m}^{-1}$ at $333 \mathrm{~K}$ and under high humid conditions, which is comparable to the $\mathrm{K}^{+}$conductivity in $\mathrm{K}^{+}$channel (Fig. 3b) [24]. The COFs derived from hexamine and tetraone demonstrated both high-water permeation $\left(\sim 2260 \mathrm{~L} \mathrm{~m}^{-2} \mathrm{~h}^{-1} \mathrm{bar}^{-1}\right)$ and a cation size selectivity performance (Fig. 3c) [25]. ZIF-8 MOF porous membrane with an average pore size of $\sim 0.34 \mathrm{~nm}$ showed a high $\mathrm{LiCl} / \mathrm{RbCl}$ selectivity of $\sim 4.6$, and ultrafast ion transport rate of $\sim 10^{6}$ ions/s (Fig. 3d) [26]. UiO-66 MOF channel with $\sim 0.6 \mathrm{~nm}$ in pore size showed ultrahigh $\mathrm{F}^{-}$conductivity $\left(\sim 10 \mathrm{~S} \mathrm{~m}^{-1}\right)$ and ultrahigh $\mathrm{F}^{-} / \mathrm{Cl}^{-}$selectivity $(\sim 240)$ (Fig. 3e) [27]. All of these subnanometer porous materials would provide artificial ion channel environment to simulate biological ion channel, which could optimize detection technology to promote the investigation of quantum-confined ion superfluid and neural signals in biological systems.

Furthermore, new information technology based on quantum ions as bioinformation carrier would be developed. Something like the emergence of $p-n$ junction transistors has generated integrated circuits and microelectronics, and led to a revolution in electronic technology (Fig. 4a). The emerging research of bionic ion channels and quantum ionics would lead to a revolution in information technology (Fig. 4b). Quantum ion integrated circuits would be superior to electronic integrated circuits, just as the human brain is superior to computers. The remaining challenges are the fabrication of soft-matter-based super ionic conductance materials


Quantum ionic diode
Quantum ion intearated circuit

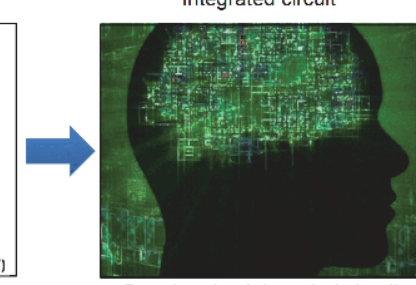

Figure 4 Future quantum ionics technology. (a) The first transistor model of p-n junction and highly developed integrated circuit. (b) The first model of quantum ionic diode and the unpredictable future information technology based on quantum ion integrated circuit. and construction of quantum-ion-based logic unit devices.

In summary, bioinformation is based on alternating current signal, and the introduction of quantum ionics concept into the biology field would provide new viewpoint and technology to study bioinformation transformation. It would not only promote the development of neural and brain science, but also generate future information technology.

Received 29 September 2019; accepted 17 October 2019; published online 30 October 2019

1 Marmont G. Studies on the axon membrane. I. A new method. J Cell Comp Physiol, 1949, 34: 351-382

2 Neher E, Sakmann B. Single-channel currents recorded from membrane of denervated frog muscle fibres. Nature, 1976, 260: 799-802

3 Campbell PK, Jones KE, Normann RA. A 100 electrode intracortical array: Structural variability. Biomed Sci Instrum, 1990, 26: $161-165$

4 Duan X, Gao R, Xie P, et al. Intracellular recordings of action potentials by an extracellular nanoscale field-effect transistor. Nat Nanotech, 2012, 7: 174-179

5 Tian B, Liu J, Dvir T, et al. Macroporous nanowire nanoelectronic scaffolds for synthetic tissues. Nat Mater, 2012, 11: 986-994

6 Park DW, Schendel AA, Mikael S, et al. Graphene-based carbonlayered electrode array technology for neural imaging and optogenetic applications. Nat Commun, 2014, 5: 5258

7 Williamson A, Ferro M, Leleux P, et al. Localized neuron stimulation with organic electrochemical transistors on delaminating depth probes. Adv Mater, 2015, 27: 4405-4410

8 Fu TM, Hong G, Zhou T, et al. Stable long-term chronic brain mapping at the single-neuron level. Nat Methods, 2016, 13: 875882

9 Hodgkin AL, Huxley AF. A quantitative description of membrane current and its application to conduction and excitation in nerve. J Physiol, 1952, 117: 500-544

10 Parker JL, Shariati NH, Karantonis DM. Electrically evoked compound action potential recording in peripheral nerves. Bioelectron Med, 2018, 1: 71-83

11 Zhang X, Jiang L. Quantum-confined ion superfluid in nerve signal transmission. Nano Res, 2019, 12: 1219-1221

12 Wen L, Zhang X, Tian Y, et al. Quantum-confined superfluid: From nature to artificial. Sci China Mater, 2018, 61: 1027-1032

13 Zhang $\mathrm{X}$, Liu H, Jiang L. Wettability and applications of nanochannels. Adv Mater, 2019, 31: 1804508

14 Hao Y, Zhang X, Jiang L. Quantum-confined superfluid. Nanoscale Horiz, 2019, 4: 1029-1036

15 Markram H. The human brain project. Sci Am, 2012, 306: 50-55

16 Ruiz-Manresa F, Grundfest H. Synaptic electrogenesis in eel electroplaques. J General Physiol, 1971, 57: 71-92 (Note: The electrocytes in the electric eels could generate high potential of $\sim 600 \mathrm{~V}$ and high current density of $500 \mathrm{~A} / \mathrm{m}^{2}$ within $20 \mathrm{~ms}$.)

17 Zhou Y, Morais-Cabral JH, Kaufman A, et al. Chemistry of ion coordination and hydration revealed by a $\mathrm{K}^{+}$channel-Fab complex at $2.0 \AA$ resolution. Nature, 2001, 414: 43-48

18 Personal communication with Prof. Markus Antonietti, Max 
Planck Institute of Colloids and Interfaces, Germany. March, 2018 19 Funkner S, Niehues G, Schmidt DA, et al. Watching the lowfrequency motions in aqueous salt solutions: The terahertz vibrational signatures of hydrated ions. J Am Chem Soc, 2012, 134: $1030-1035$

20 Liu G. The conjectures on physical mechanism of vertebrate nervous system. Chin Sci Bull, 2018, 63: 3864-3865

21 Schmidt DA, Birer O, Funkner S, et al. Rattling in the cage: Ions as probes of sub-picosecond water network dynamics. J Am Chem Soc, 2009, 131: 18512-18517

22 Kratochvil HT, Carr JK, Matulef K, et al. Instantaneous ion configurations in the $\mathrm{K}^{+}$ion channel selectivity filter revealed by $2 \mathrm{D}$ IR spectroscopy. Science, 2016, 353: 1040-1044

23 Blanchard F, Doi A, Tanaka T, et al. Real-time terahertz near-field microscope. Opt Express, 2011, 19: 8277-8284

24 Xing G, Yan T, Das S, et al. Synthesis of crystalline porous organic salts with high proton conductivity. Angew Chem Int Ed, 2018, 57: 5345-5349

25 Kuehl VA, Yin J, Duong PHH, et al. A highly ordered nanoporous, two-dimensional covalent organic framework with modifiable pores, and its application in water purification and ion sieving. J Am Chem Soc, 2018, 140: 18200-18207

26 Zhang H, Hou J, Hu Y, et al. Ultrafast selective transport of alkali metal ions in metal organic frameworks with subnanometer pores. Sci Adv, 2018, 4: eaaq0066

27 Li X, Zhang $\mathrm{H}$, Wang $\mathrm{P}$, et al. Fast and selective fluoride ion conduction in sub-1-nanometer metal-organic framework channels. Nat Commun, 2019, 10: 2490

28 MacKinnon R. Potassium channels and the atomic basis of selective ion conduction (Nobel lecture). Angew Chem Int Ed, 2004, 43: $4265-4277$

Acknowledgements This work was supported by the National Key R\&D program of China (2016YFA0200803), and the National Natural Science Foundation (51973227 and 51603211).

Author contributions Jiang L proposed the concept of quantum ionics. Antonietti $\mathrm{M}$ suggested the absorption of ion in $\mathrm{THz}$ range. Zhang $\mathrm{X}$ and Jiang $\mathrm{L}$ wrote the paper and contributed to the general discussion.

Conflict of interest The authors declare that they have no conflict of interest.

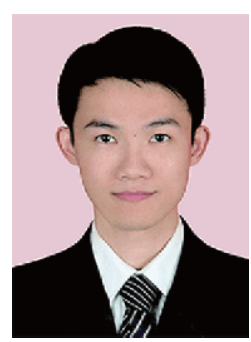

Xiqi Zhang is an associate professor in Prof. Lei Jiang's group at the Technical Institute of Physics and Chemistry (TIPC), Chinese Academy of Sciences. He received his BSc degree (2007), MSc degree (2009) and PhD (2012) from Sun Yat-sen University under the supervision of Prof. Jiarui $\mathrm{Xu}$ and Zhenguo Chi. Afterwards, he worked as a post-doctoral fellow with Prof. Yen Wei at Tsinghua University. In 2014, he joined TIPC. His current scientific interests are quantumconfined superfluid and its application.

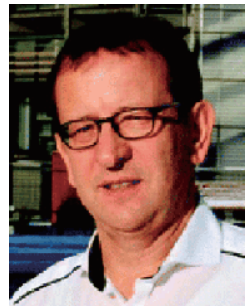

Markus Antonietti is a Professor at the Max Planck Institute (MPI) of Colloids and Interfaces. He studied Chemistry in Mainz, where he also received his $\mathrm{PhD}$ in 1985 . His habilitation on nanogels in 1990 fueled his enthusiasm for complex nanostructures based on polymers and carbon. After a professorship at the University of Marburg, he was appointed director for the department of Colloid Chemistry at the MPI of Colloids and Interfaces in 1993. His work deals with modern materials chemistry, energy materials, and sustainability issues within those topics.



Lei Jiang is a Professor at the TIPC and Beihang University. He is an academician of the Chinese Academy of Sciences, Academy of Sciences for the Developing World, and National Academy of Engineering, USA. He received his Bachelor's and Master's degrees from Jilin University, and $\mathrm{PhD}$ from the University of Tokyo. He worked as a post-doctoral fellow with Prof. Akira Fujishima and then as a senior researcher in the Kanagawa Academy of Sciences and Technology with Prof. Kazuhito Hashimoto. In 1999, he joined the Institute of Chemistry, Chinese Academy of Sciences as part of the Hundred Talents Program. In 2015, he and his group moved to TIPC. His scientific interests focus on bio-inspired, smart, multi-scale interfacial materials with superwettability.

\section{生物信息转化: 从离子学到量子离子学}

张锡奇 ${ }^{1}$, Markus Antonietti ${ }^{4}$, 江雷 ${ }^{1,2,3 *}$

摘要 传统的神经记录技术是基于从离子学到电子学的生物信息 转换, 虽被广泛研究, 但其在神经科学和脑科学领域进展很小. 最 近, 生物离子通道中的离子和分子流动被看作是量子限域超流体, 即离子和分子的量子态可作为生物信息载体, 其吸收光谱在太赫 兹范围内, 因此太赫兹光可以作为一个工具来实现生物信号的非 接触检测. 我们提出两种研究方案: 一种是利用太赫兹响应研究生 物体系的神经信号, 另一种是利用太赫兹响应研究人工体系的量 子限域离子超流体, 并为生物体系中神经信号的检测提供优化参 数. 把量子离子学引入生物信息学领域, 将为神经信号研究提供一 个新的技术手段, 推动神经科学和脑科学的发展, 并发展量子离子 学技术. 